NASA Technical Memorandum 113109

110.35
$06 \% 085$

\title{
Achromatic Emission Velocity Measurements in Luminous Flows
}

\author{
S.J. Schneider \\ Lewis Research Center \\ Cleveland, Ohio
}

S.F. Fulghum and P.S. Rostler

Science Research Laboratory, Inc.

Somerville, Massachusetts

Prepared for the

33rd Joint Propulsion Conference and Exhibit

cosponsored by AIAA, ASME, SAE, and ASEE

Seattle, Washington, July 6-9, 1997

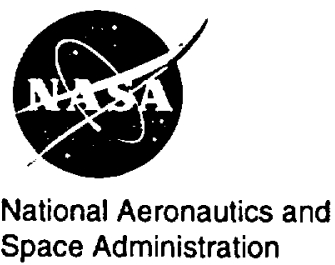




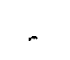

.

. 
Achromatic Emission Velocity Measurements in Luminous Flows

\author{
S. J. Schneider \\ NASA Lewis Research Center \\ Cleveland, Ohio \\ S. F. Fulghum and P. S. Rostler \\ Science Research Laboratory, Inc. \\ Somerville, Massachusetts
}

\begin{abstract}
A new velocity measurement instrument for luminous flows was developed by Science Research Laboratory for NASA. The SIEVE (Segmented Image Emission VElocimeter) instrument uses broadband light emitted by the flow for the velocity measurement. This differs from other velocimetry techniques in that it does not depend on laser illumination and/or light scattering from particles in the flow. The SIEVE is a passive, non-intrusive diagnostic. By moving and adjusting the imaging optics, the SIEVE can provide three-dimensional mapping of a flow field and determine turbulence scale size. A SIEVE instrument was demonstrated on an illuminated rotating disk to evaluate instrument response and noise and on an oxy-acetylene torch to measure flame velocities. The luminous flow in rocket combustors and plumes is an ideal subject for the SIEVE velocity measurement technique.
\end{abstract}

Aerospace Engineer, Senior Member AlAA

\section{Introduction}

A passive, non-intrusive optical instrument was developed for the measurement of localized flow velocity in luminous flows. The instrument, named SIEVE for Segmented Image Emission VElocimeter, was developed by Science Research Laboratory (SRL) under a NASA Small Business Innovative Research contract. ${ }^{1.2}$ The SIEVE diagnostic differs from other velocimetry techniques in that it uses broadband light emission from the flowing medium for the velocity measurement. The technique does not depend on light scattering from particles, so seeding or laser illumination of the flow is not required.

The operating theory for the SIEVE diagnostic is based on an interferometric technique developed for plasma diagnostics in the 1970's. ${ }^{3.45}$ The principle of operation of the SIEVE is illustrated in Figure 1. A luminous flow field is imaged onto two binary transmission gratings, which cut the images into strips or segments. Each of the gratings block half of the imaged light segments. The blocked and transmitted image segments from each grating are out of phase by $180^{\circ}$. Pockets of luminous gases sweeping through the image field are first seen on one grating and then the other and are 
sized by the spacing of the grating. The signals from these gratings are differenced to remove common mode light fluctuations. The differenced signal has a peak frequency which is proportional to the flow velocity. A similar method was used to measure the velocity of an externally illuminated liquid flow seeded with particulates in the 1960's using a single transmission grating ${ }^{6}$. The SIEVE diagnostic, however, uses two complementary gratings and differencing electronics to achieve a high degree of common mode rejection, allowing small fluctuations in the emitted light to be resolved. The imaging optics localize the velocity measurement to a volume defined by the optical aperture and depth of field. The focal volume, then, can be adjusted to provide three dimensional mapping of the flow field. Furthermore, the spatial size can be adjusted so that turbulence scales can be determined. It is important to note that since velocity measurement is dependent on fluctuations in the flow, the SIEVE diagnostic is limited to turbulent, luminous flows.

This paper gives a description of the SIEVE instrument components and data acquisition and processing. The paper also discusses testing at SRL, where a rotating disk was used to evaluate instrument response and system noise and where an oxy-acetylene torch was used for flame velocity measurements. Finally, the paper discusses the applicability of the SIEVE for measuring luminous rocket combustor flows and plumes.

\section{Instrument Description}

The instrument consists of a telescope assembly for imaging the flow field, a detector module for optically and electronically processing the image, and data acquisition and processing hardware and software. Schematics of the telescope assembly and detector module are shown in Figure 2. Each component is described below.

\section{Telescope Assembly}

The telescope system is totally achromatic and is based on the classic Schwarzschild mirror objective ${ }^{7}$ as shown schematically in Figure 2. Each mirror is coated with a protected silver coating for high, broadband reflectivity. The magnification range is from $5 \mathrm{X}$ to $20 \mathrm{X}$ and is measured by placing an object of known size at the object position and measuring the size of its image with the reticle in the SIEVE detector head. The distance from the front of the telescope housing to the object plane is approximately $160 \mathrm{~mm}$, which allows the viewing of combustion processes through windows.

\section{Detector Module}

A photograph of the detector module is shown in Figure 3. This module is split into two sections, the upper section houses the SIEVE detection optics and the lower section houses the detection electronics. The detection optics include a 3-cube beamsplitter, transmission gratings, aspherical collection lenses, focusing reticle, and an eyepiece. The detection electronics include the two silicon avalanche photodiodes, amplifiers, a differencing circuit, and a summing circuit. The sum and difference signals generated by the detector module have relatively high frequency components and are passed to a high speed digitizer, stored, and analyzed for frequency content with a fast Fourier 
transform (FFT) on a personal computer. A brief discussion of these components follows. More details on the design can be found in Reference 2 .

The 3-cube beamsplitter ensures that each photodetector sees the same image of the object in terms of both wavelength and intensity. The beamsplitter cubes used in the SIEVE are optimized for $50 \%$ transmission and reflection for wavelengths between $700 \mathrm{~nm}$ and 1100 $\mathrm{nm}$. Shorter wavelengths are predominantly transmitted and longer wavelengths are predominantly reflected. Because each detector sees an image that has been both reflected and transmitted, the images are as identical as the beamsplitters.

The two fixed transmission gratings are located in the image planes of the telescope. They are $20 \mathrm{~mm}$ by $20 \mathrm{~mm}$ square and set the optical aperture of the instrument. The transmission gratings transmit a binary pattern of light and dark fringes to each image from the beamsplitter. These fringes are $180^{\circ}$ out of phase on each image. The mirror system on the telescope projects the optical aperture onto the flow field to set the detection area at from $1 \mathrm{~mm}$ by 1 $\mathrm{mm}$ to $4 \mathrm{~mm}$ by $4 \mathrm{~mm}$ depending on the magnification. The period on the grating can be changed by installing a different grating. As discussed later, the instrument sensitivity peaks when the grating period is twice the size of characteristic dimensions of disturbances in the flow. This allows measurement of turbulence scale as well as velocity. Instrument sensitivity is also peaked in the object plane, but the derived signal has contributions from disturbances within the depth of field. The depth of field can be shown from geometric optics to be proportional to the diameter of the detection area and the fringe spacing.

The SIEVE photodetectors are silicon avalanche photodiodes (APD), which work well in the near infrared. The current from each APD is converted to a voltage with a wide-band amplifier in a transimpedence configuration. These voltages are then differenced with a wide-band amplifier and passed to the DC Difference output (DCD visible in Figure 3 ). The DC difference signal is balanced during setup for common mode rejection of source intensity fluctuations. Balancing the difference signal requires that one of the bias voltages (and thus the photodetector gain) be reduced from its maximum position until the DC difference signal is zero. The two voltages are also summed with an opamp and passed to the DC Sum output (DCS visible in Figure 3) in the same way. The DC Sum signal is monitored to assure that the photodiode's signals are within their linear limits. It is also used to determine low frequency noise due to an imperfectly balanced bridge circuit coupled with large optical power fluctuations in the source. The AC difference output $(A C D$ visible in Figure 3 ) is provided by a circuit which filters off both DC offsets and AC signals below about $1 \mathrm{kHz}$ to prevent these signals from compromising the dynamic range of the signal digitizer. This output is the primary output from the SIEVE detector module. The useful bandwidth of the circuit ranges from $1 \mathrm{kHz}$ to about $30 \mathrm{MHz}$. The output noise from the circuit is quite low in the band between 1 $\mathrm{kHz}$ and $5 \mathrm{MHz}$, (limited by the transient 
digitizer), where many SIEVE signals can be generated.

\section{Data Acquisition and Processing} The SIEVE instrument has a 12-bit, 2channel transient digitizer with a 1 megasample (MS) memory depth (1024 $X$ 1024) and a maximum data acquisition rate of $20 \mathrm{MS} / \mathrm{s}$ on one channel and $10 \mathrm{MS} / \mathrm{s}$ when both channels are used (as is typically the case). Two input channels connected to the $\mathrm{AC}$ difference and $\mathrm{DC}$ sum signals from the detector module are simultaneously digitized and stored. A fast Fourier transform (FFT) is used to determine the peak frequency in the fluctuating difference signal. The peaks produced by the SIEVE with light from turbulent flows are relatively broad (typically about $1 / 3$ to $1 / 7$ of the center frequency), so relatively few FFT points are required to resolve them. The optimal analysis uses the longest possible data set taken at the lowest possible sampling rate. The data set is then divided into subintervals during processing and the FFT's of the subintervals are averaged to obtain the final frequency spectrum. A parabolic curve fit to the frequency peak provides the final estimate of its central frequency (and thus the flow velocity).

\section{Instrument Demonstration}

Operation of the instrument was verified at SRL on a rotating disk with background illumination and on an oxyacetylene torch. Results obtained from each device are discussed below along with an evaluation of instrument noise.

Rotating Disk Experiments

A rotating disk was chosen for evaluation by the SIEVE because of its known velocity and its planar scattering surface, which was well suited to probing the depth of field response of the instrument. The disk consists of a rotating, transparent wheel, illuminated from behind, with its front surface slightly pitted to scatter light. An $0.2 \mathrm{~m}$ diameter, Plexiglass scattering wheel mounted on a machined hub attached to an $1800 \mathrm{rpm}$ synchronous motor was used. Near the rim of the wheel the velocity was about $15 \mathrm{~m} \mathrm{~s}^{-1}$, which corresponds to a SIEVE signal with a central frequency of about $150 \mathrm{kHz}$, when used with a $20 \mathrm{X}$ magnification and a $2 \mathrm{~mm}$ period grating. A motorized, encoded, $X-Y$ translation stage was used to move the scattering surface of the rotating wheel back and forth along the optical axis and through the focal volume of the magnification optics. In this way, the instrument response function could be determined for a given set of transmission gratings.

Figure 4 shows a scan of the instrument depth of field response function taken with the 5-cycle, $4 \mathrm{~mm}$ period transmission gratings and the Schwarzschild magnifier set to $20 \mathrm{X}$. The rotating wheel had surface pits similar to point sources. The depth of field for this case is determined from geometric optics to be approximately 9 $\mathrm{mm}$. Figure 4 verifies this depth of field by showing the response function is small at the $+1-4.5 \mathrm{~mm}$ points on the curve and shows that points outside of this range would have little contribution to the final data spectrum. The minima at $+/-0.8 \mathrm{~mm}$ are the points at which the defocused spot of a point source equals the period of the grating at the SIEVE detection head as shown in Figure 5. The second peak at $+/-1.2 \mathrm{~mm}$ is where the 
spot size equals 1.5 grating periods. Typically, however, the sources in a SIEVE image are not well defined points. Broad scratches on the wheel or flame turbulence, have a range of sizes so that the minima and secondary maxima of Figure 4 do not appear in more typical measurements.

Figure 6 shows SIEVE depth-of-field scans of a more typical image taken with three different grating periods at $20 \mathrm{X}$ magnification. As expected, the grating with the smallest period provided the best resolution along the optical axis since its depth of field is $+/-2.2 \mathrm{~mm}$. This is further shown in Figure 7 where the peaks of the response functions of the $2 \mathrm{~mm}$ and $4 \mathrm{~mm}$ gratings are normalized to the same value.

There is a straightforward relationship between the instrument response as a function of defocus and the instrument response as a function of source size (image spatial scale size) in the focal plane. Sources whose images have a characteristic size equal to about half the grating period or less give the strongest signal. Sources with image sizes equal to the grating period produce no signal. Sources with large or very small image sizes compared to the grating period produce small signals.

Increasing the number of cycles in the transmission grating (besides decreasing the SIEVE depth-of-field) increases the center frequency of the SIEVE signal and decreases its relative width. These effects are shown in Figure 8 which plots analyses of $2 \mathrm{~mm}, 2.5 \mathrm{~mm}$, and $4 \mathrm{~mm}$ grating data sets obtained in the depthof-field scans of Figure 6 . The width of the SIEVE signal peak is inversely proportional to the transit time of an image point across the $20 \mathrm{~mm}$ aperture of the detection head. The grating period (width of one cycle) sets the central frequency. Thus, the ratio of the central frequency to width of the peak is the number of grating cycles. This trend is shown in Figure 8 in which the measured ratio is less than the actual number of cycles on the grating, as expected, since the gratings are apodized, effectively reducing the number of cycles, to minimize higher harmonic generation.

Figure 9 shows several of the individual FFT's of the data points from the scan shown in Figure 4. The frequency interval (bandwidth $B$ ) in these plots is $1220.7 \mathrm{~Hz}$. This interval is important because the system noise power, as shown in the plot, including thermal noise and shot noise, is proportional to this value.

Figure 9 (a) shows an FFT of a SIEVE signal taken with the optics focused directly on the scattering surface of the rotating wheel. In this case the SIEVE signal is much larger than any of the noise sources and is easily identified and evaluated for its central frequency and thus the velocity.

In order to evaluate a low signal to noise ratio measurement with the rotating disk, the SIEVE signal is measured with the rotating disk outside of the $+/-4.5 \mathrm{~mm}$ depth of field $(6 \mathrm{~mm}$ in front of the focal point) of the Schwarzschild magnifier. This FFT is shown in Figure 9 (b). The peak of this signal is very small (about $1 \%$ of the peak at the best focus) and the background noise is significant. The most obvious noise is the low frequency 
peak (which is due to slight imbalances between the APD gains and optical transmissions), coupled with fluctuations in the overall intensity of the moving source (large scale variations in the amount of light scattered from the rotating wheel at different points around its rim). Much of this peak can be removed in data processing, if it overlaps the desired signal frequency, by subtracting a fraction of the sum signal from the difference signal. That is not the case here and has not been done in this particular analysis. The SIEVE peak is well separated for analysis.

In both Figures 9 (a) and 9 (b), the background level of the FFT was subtracted from the data during analysis to provide a more accurate measurement of the amplitude of the SIEVE signal peak. In the FFT's shown in Figures 9 (c) and 9 (d) the background was not subtracted so that it could be compared to theoretical predictions.

Figure 9 (c) shows the FFT of a data set taken with the rotating wheel stopped so that there would be no SIEVE signal. The illumination source was kept on, however, to measure the level of noise inherent in the optical detection part of the SIEVE system. The background noise level in 9 (c) is flat over the measured frequency interval as would be expected for thermal and shot noise sources. The two sharp peaks at $65 \mathrm{kHz}$ are due to residual noise in the detection circuit power supply and are easily distinguished from true SIEVE signals by the fact that they are so narrow.

Figure 9 (d) shows an FFT of the system noise with the incoming light blocked, so that only the electronics noise in the detection circuits and the digitizing circuits is present. Again, the $65 \mathrm{kHz}$ noise from the power supply is evident. The largest source of background noise at these frequencies is the two, $5000 \Omega$, load resistors in the transimpedance amplifiers, which convert the APD photocurrent to a signal voltage. The signal, however, is proportional to this resistance, while the noise is only proportional to the square root of this resistance.

A detailed analysis of the noise in these plots is presented in Reference 2 . The fact that these noise levels can be predicted reasonably well means that the ability of the SIEVE to perform a measurement in a particular circumstance can also be predicted. The difficulty will always be estimating the level of optical fluctuations which might be present at the scale size of interest.

\section{Oxy-acetylene Torch}

An oxy-acetylene torch was chosen as a simple, turbulent, combusting flow field to demonstrate the SIEVE's operation.

The torch was also convenient to demonstrate the effect of instrument depth-of-field broadening on a velocity scan along the optical axis compared to a velocity scan transverse to the optical axis. The torch was mounted vertically on an X-Y translator in the horizontal plane with the optical axis of the magnifier in the horizontal plane. All of the SIEVE measurements were thus taken in a single transverse cross section of the torch flame. Figure 10 shows two CCD images of a typical flame used for these tests. The bottom image has eight times the exposure of the top image to better show the less luminous region, 
where the flow is laminar. The camera was set for an exposure of $100 \mu$ s which, at a typical flow velocity for an oxyacetylene torch, of $25 \mathrm{~m} \mathrm{~s}^{-1}$, means that structure below a scale size of about 2.5 $\mathrm{mm}$ is washed out. Several axial locations of the flow were tested for signal-to-noise ratio and a plane was selected for measurements about $1 \mathrm{~cm}$ beyond the point where the flow becomes turbulent.

The FFT's of some SIEVE data collected in this plane are presented in Figure 11. The magnification was 20X, so the cross section of the probe volume was $1 \times 1 \mathrm{~mm}$. The grating was $4 \mathrm{~mm}$ giving a $9 \mathrm{~mm}$ depth of field for point sources in the flow. The effective grating period projected onto the flame was only $200 \mu \mathrm{m}$. This is smaller than any structure shown in the images in Figure 10. These images, therefore, do not show the flow structure which generates the SIEVE signal. It is also likely that most of the power in the optical fluctuations which generate the signal is at much larger scale sizes than $200 \mu \mathrm{m}$. Consequently, the SIEVE signals shown in Figure 11 are relatively small compared to those of the more ideal scattering wheel tests in Figure 9. Also, note that in the scattering wheel tests, the smaller grating periods yielded smaller signals. This is even more likely to be the case with flow signals generated by turbulence, because signal power falls off rapidly with decreasing scale size, due to thermal diffusion limits in the flow. Another effect expected in SIEVE diagnostics of turbulent flows is that the width of the SIEVE frequency peak is broader than indicated by the number of grating cycles. This indicates that sources of the signal, regions of greater or lesser luminosity, do not survive more than a few characteristic diameters. A larger number of grating cycles, therefore, may not decrease the width of the SIEVE frequency signal peak in these cases. These considerations suggested that the $4 \mathrm{~mm}$ grating was the appropriate choice.

The flame was centered on the detector by placing a fine tungsten wire tip at the optical focus of the magnifier. A nonturbulent, blue flame was generated and centered on that tungsten tip. The acetylene flow was then increased until the flame became turbulent and luminous (possibly sooting). This allignment procedure was not very satisfactory, however. When the flame was luminous, its transverse position still required adjustment so that its image was centered on the detector head aperture. The position of the flame along the axis could not be easily checked.

The SIEVE data were first taken at the expected center of the flame. The FFT of this data is presented in Figure 11 (a). The SIEVE velocity peak in this data is shown to be very small compared to the low-frequency noise peak generated by fluctuations in the overall intensity of the flame. Figure 11 (b) is the same FFT data scaled to show the SIEVE peak. The raw data for this graph consists of $512 \mathrm{KSamples}$ of data taken at $500 \mathrm{KS} / \mathrm{s}$ for a total run duration of 1.05 seconds. The upper half of the peak (above the half-maximum) of the data in Figure 11 (b) is easily analyzed with a parabolic curve fit and gives a center frequency which indicates an average flame velocity of $25 \mathrm{~m} / \mathrm{s}$ over the 1 second run. No comparable measurement was found 
in the literature. The centerline velocity in a propane/air flame was measured at $3.17 \mathrm{~m} / \mathrm{s}$ with a laser Doppler velocimeter ${ }^{8}$. Acetylene/air was reported to have a laminar flame velocity approximately four times that of propane in air?. The influence of oxygen concentration on flame speed was reported to be a factor of eight higher for propane/oxygen over propane/air ${ }^{10}$. Thus, the data are in the range likely for this type of flow. Figure 11 (c) shows FFT data taken with the flame moved 3 $\mathrm{mm}$ horizontally so that the measurement was at the very edge of the luminous portion of the flame. Here the total light collected by the objective is smaller and the curve fit to the SIEVE peak indicates that the velocity has dropped to $15.0 \mathrm{~m} / \mathrm{s}$. Note that in this case, the SIEVE is not looking through regions where the flame is more luminous and moving faster. Figure 11 (d) shows a FFT data set taken with the flame moved $2 \mathrm{~mm}$ back along the optical axis behind the focal point of the optics. The magnifier is thus focused on the front edge of the flame with the light from the rest of the flame still entering the detection head. The velocity in this case is measured to be $18.7 \mathrm{~m} / \mathrm{s}$, which is consistent since this point is closer to the flame core.

The axial and transverse velocity profiles of the turbulent oxy-acetylene flame are shown in Figure 12. Data taken with the flame closer to the detection head (in front of the focal point of the optics) indicates a higher velocity than the centerline. The SIEVE signal from flows in front of and behind the focal point should give the same velocity unless the flow is optically thick or so turbulent that it distorts the view of the flow behind. Neither of these scenarios appears to be the case. An experiment was performed to test the optical distortion of the flame by focusing a diffraction-limited $\mathrm{HeNe}$ laser beam onto the core of the flame with a fast lens and collecting the light that passed through the flame with the same, large Schwarzschild objective at the same $20 \mathrm{X}$ magnification setting. This test gives twice the optical distortion that a point source would pick up on a single pass out from the center of the flame. No significant distortion of the focal point image was observed at the position of the detection head. The phase distortions due to the flame are not sufficient to turn into intensity changes over a distance of a few millimeters. The most likely explanation is that measurements of velocity near the front and back edge are indeed affected by the brighter, faster core flow and that the flame was actually centered about $2 \mathrm{~mm}$ closer to the detector head than expected.

\section{Assessment of Applicability to} Rocket Flows

Based on the successful tests with the oxy-acetylene torch, the SIEVE diagnostic is expected to find application as a non-intrusive flow diagnostic for luminous flows in combustors and chemical rocket engines. The transient digitizer used in these experiments limits the measurable upper range of the flow to around Mach 2, with $2 \mathrm{~mm}$ lateral resolution and $12 \mathrm{~mm}$ depth of field. This should yield useful measurements in small rockets and laboratory apparatus. Higher Mach numbers can be measured with less spatial resolution. For example, Mach 20 can be measured with $2 \mathrm{~cm}$ lateral resolution and $12 \mathrm{~cm}$ 
depth of field. This measurement may be useful for large rockets and scramjets.

Because the SIEVE does not require active laser illumination or precise allignment, it is relatively straightforward to use. Expected SIEVE signal levels can be predicted given an estimate of the scale size and magnitude of optical fluctuations. Measured spectra from rocket engine exhausts typically show a great deal of line structure superimposed on a continuous background. This is fortunate since a flame with a blackbody spectrum would be opague and the center of the flow would not be visible. Initial efforts to take velocity measurements in a twodimensional, optically accessible rocket chamber were not successful. This is likely due to the scale size of the turbulent fluctuations being too large for the detection system and to the fact that the hydrogen-oxygen engine studied is not sufficiently luminous. A lower magnification and longer signal record lengths might increase the signal and lower the noise respectively.

\section{Conclusions}

A new velocity measurement diagnostic for luminous flows was demonstrated in a laboratory environment at Science Research Laboratory. A commercial prototype of the instrument is presently undergoing evaluation at NASA. A detailed description of the optical and data acquisition systems is provided. The diagnostic is shown to work on scattering media such as scratches on the surface of a transparent rotating disk and on a luminous (possibly sooting) oxyacetylene torch. Estimates suggest that the diagnostic will be of value in determining local velocities in the turbulent, luminous flows of rockets and combustors. The diagnostic will be further tested at NASA to determine the instrument's capabilities and limitations.

\section{$\underline{\text { References }}$}

1. Fulghum, S. F., "Multiple-Beam Spectroscopy for Liquid Rocket Engine Diagnostics", SRL-7-F-1992, Science Research Laboratory, NASA Contract NAS3-26332, July, 1992.

2. Fulghum, S. F. and Rostler, P. S., "Multiple-Beam Spectroscopy for Liquid Rocket Engine Diagnostics", SRL 06-F-1996, Science Research Laboratory, NASA Contract NAS3-27001, August, 1996.

3. Rostler, P., Cooper, W., and Kunkel, W., "Multiple-Beam Spectroscopy", Bull. Amer. Phys. Soc., 17, 986 (1972).

4. Rostler, P., "Multiple-Beam Spectroscopy", (Ph.D. Thesis) Lawrence Berkeley Laboratory Report LBL-2014 (May 1974).

5. Billard, B., "Evaluation of Two-Beam Spectroscopy as a Plasma Diagnostic", (Ph.D. Thesis) Lawrence Berkeley Laboratory Report LBL-10913 (1980). 
6. Gaster, M., "A new technique for the measurement of low fluid velocities," J. Fluid Mech., 20, 183-192 (1964).

7. Wetherell, W. and Rimmer, M., "General Analysis of Aplanatic Cassegrain, Gregorian, and Schwarzschild Telescopes," Appl. Opt. 11 (12), 2817-2832 (1972).

8. Larsen, P., Kristensen, H., and Borgnakke, C., "Bunsen Flame Velocity Distributions by LDA," Proceedings of the LDA-Symposium Copenhagen, 663673 (1975).

9. Reynolds, T. And Gerstein, M., "Influence of Molecular Structure of Hydrocarbons on Rate of Flame Propagation," Third Symposium (International) on Combustion, 190-194 (1949).

10. Zabetakis, M., "Flammability Characteristics of Combustible Gases and Vapors," U.S. Bureau of Mines Bulletin No. 627, (1965). 
A FLOWING, LUMINOUS, NON-UNIFORM GAS OR PLASMA

WILL HAVE SMALL VOLUMES WHICH EMIT

MORE OR LESS LIGHT THAN THE AVERAGE

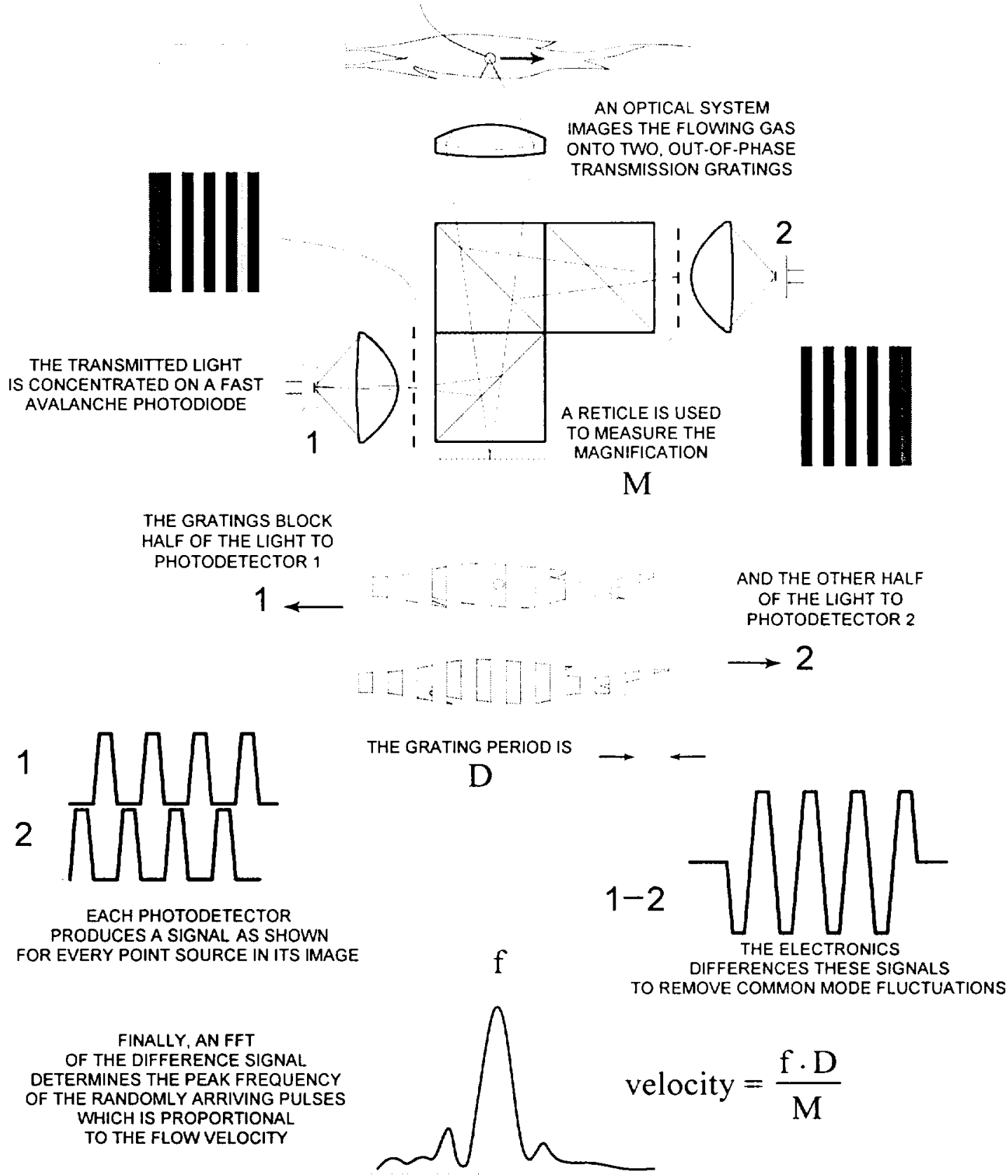

Figure 1. Schematic depiction of the principles of operation of the SIEVE. 


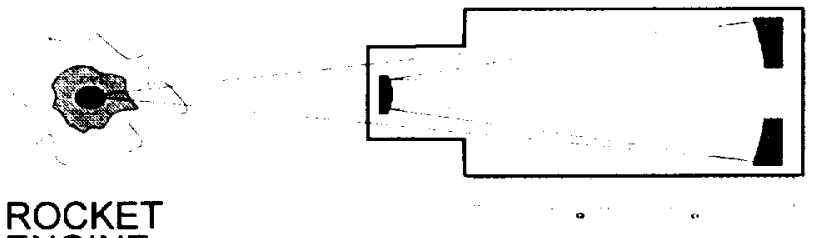

ENGINE

LIGN3.DS4
TELESCOPE ASSEMBLY
OPTICAL HEAD

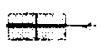

ELECTRONICS

RAIL

DETECTOR MODULE

Figure 2. Schematic of Instrument Setup.

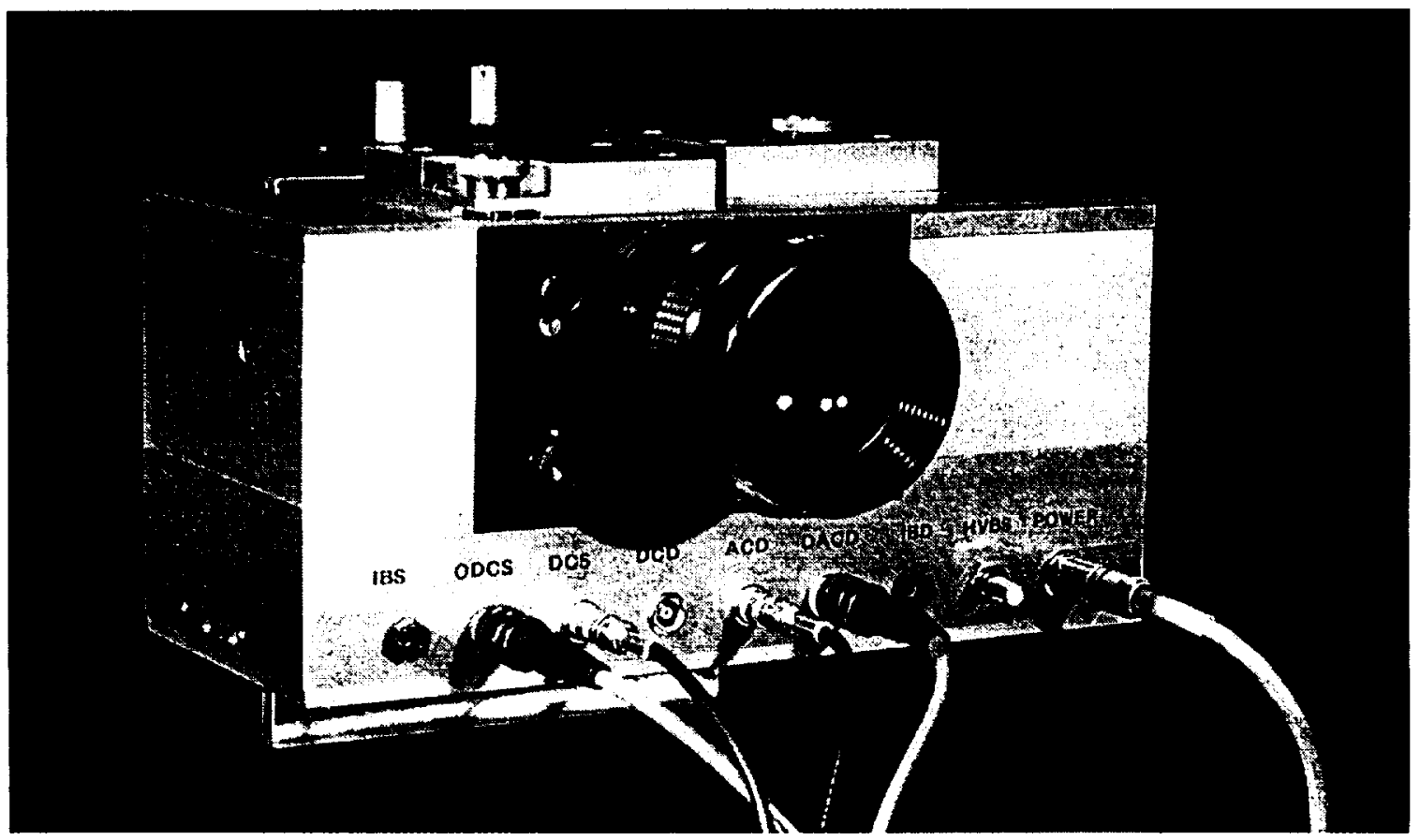

Figure 3. Photograph of Detector Module. 


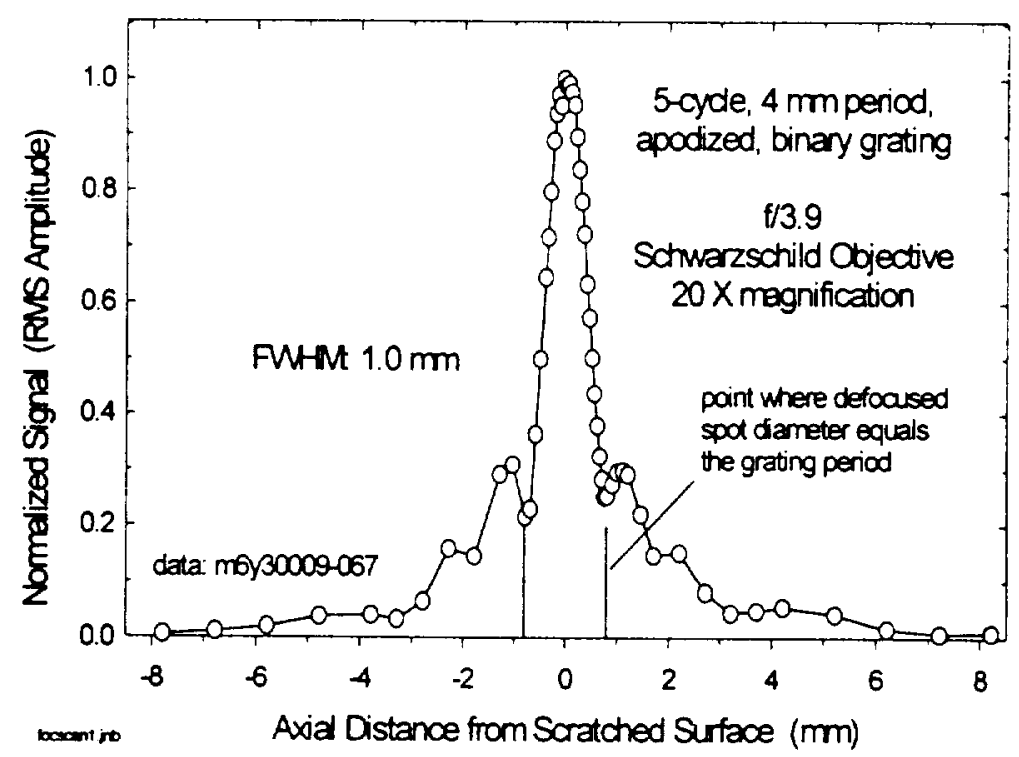

Figure 4. Measured SIEVE Instrument Response Function.

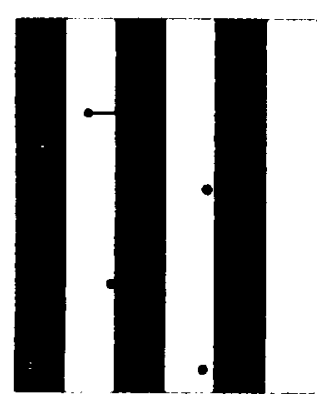

PEAK SIGNAL AT FOCUS

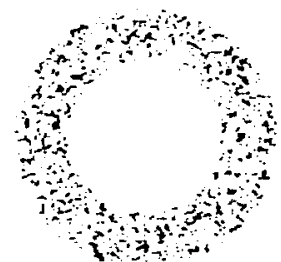

Y -TRACED OUT-OF-FOCUS SPOT FOR THE LARGE SCHWARZSCHILD MAGNIFIER

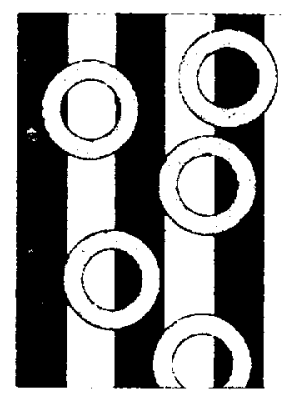

FIRST MINIMUM WHERE SPOT SIZE EQUALS GRATING PERIOD

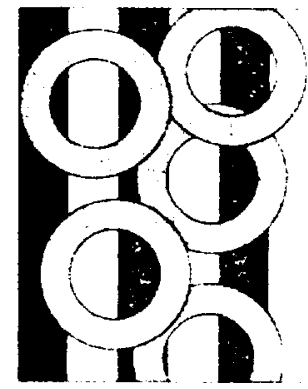

SECOND PEAK WHERE SPOT SIZE EQUALS 1.5 PERIODS

Figure 5. Illustration of spot size in the image plane due to a point source in the object plane and its relationship to the SIEVE response function. 


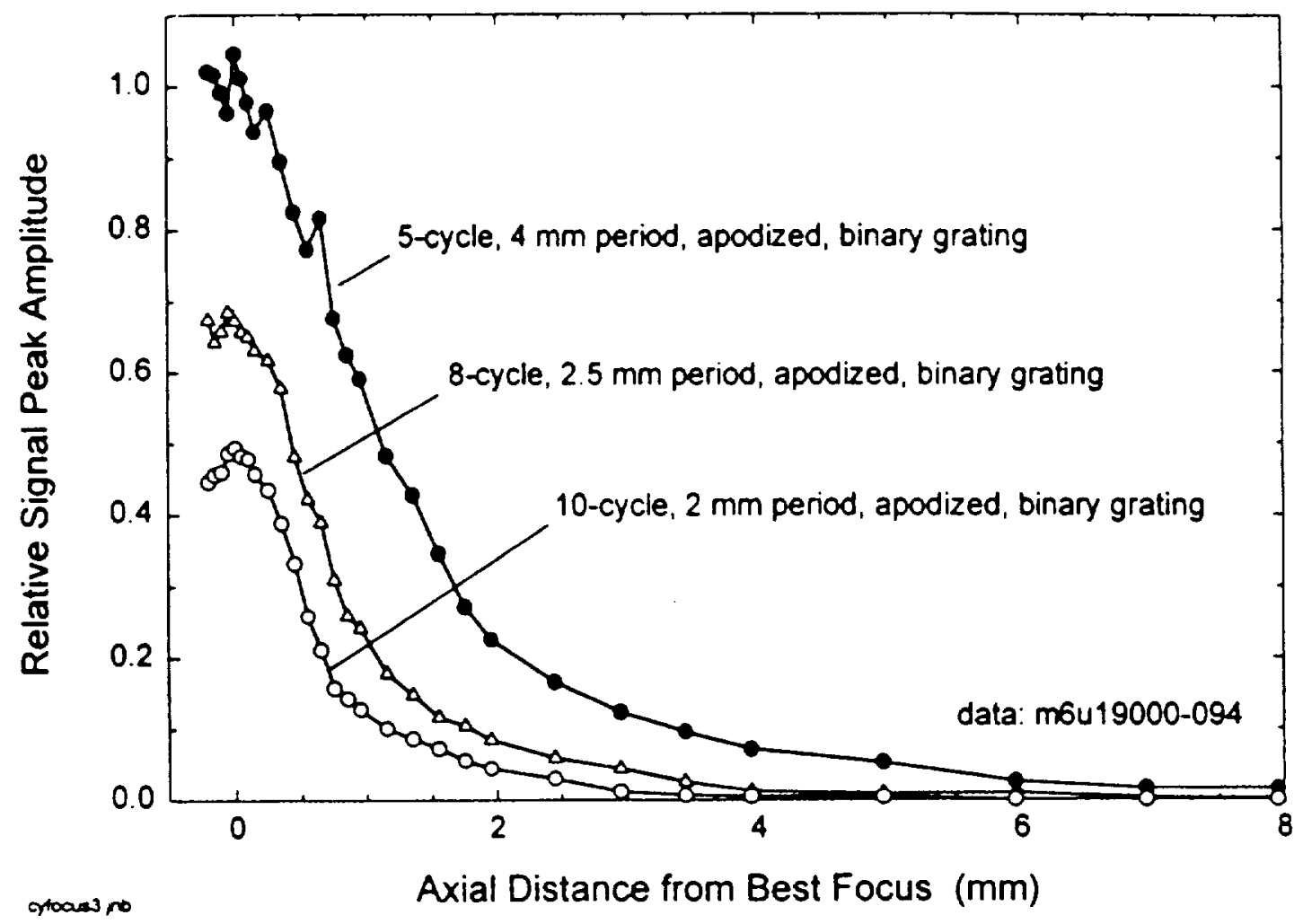

Figure 6. SIEVE depth-of-field response versus grating period.

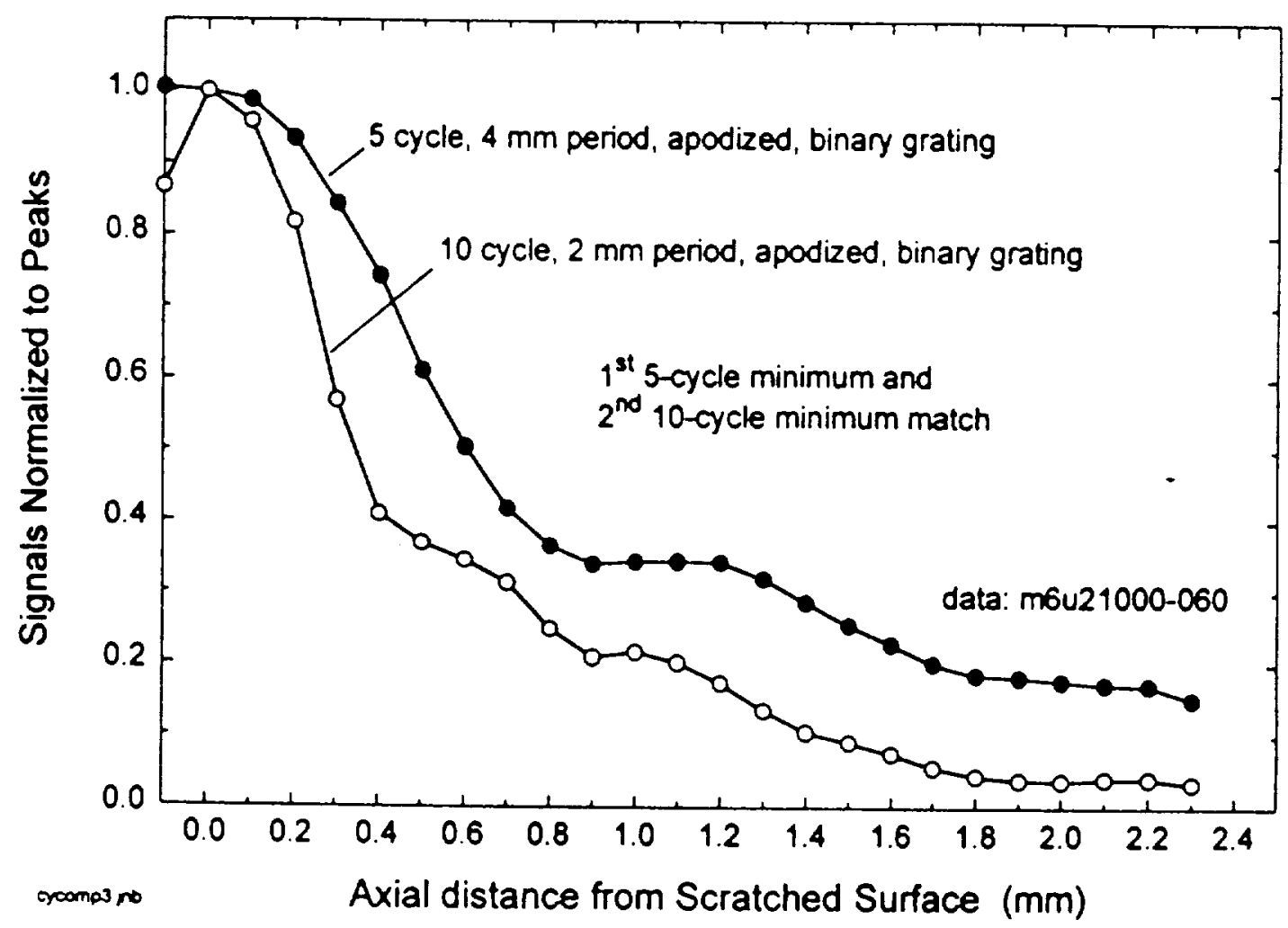

Figure 7. Normalized SIEVE depth-of-field response for 2 and $4 \mathrm{~mm}$ gratings. 

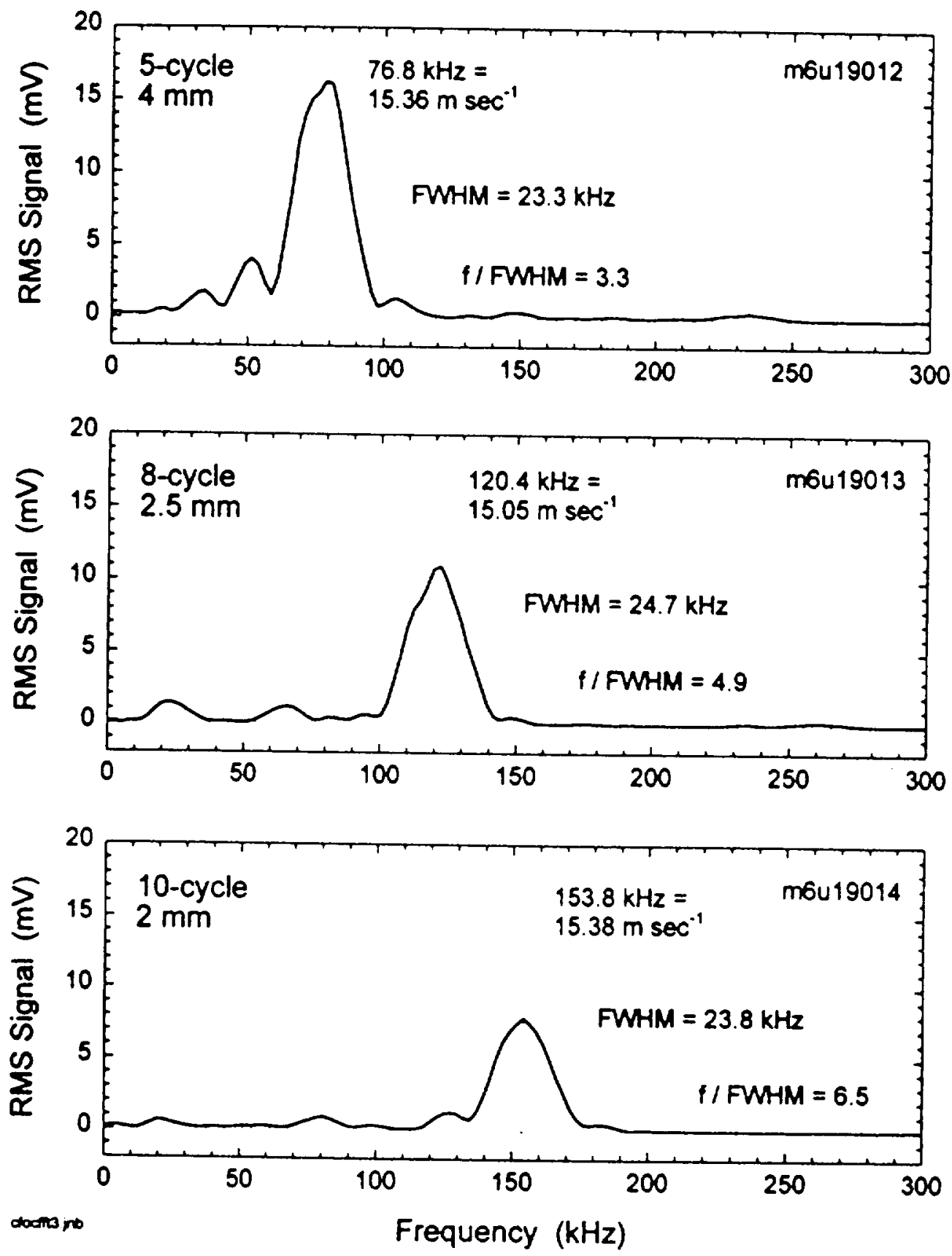

Figure 8. FFT"S of SIEVE signals from the rotating wheel source. 

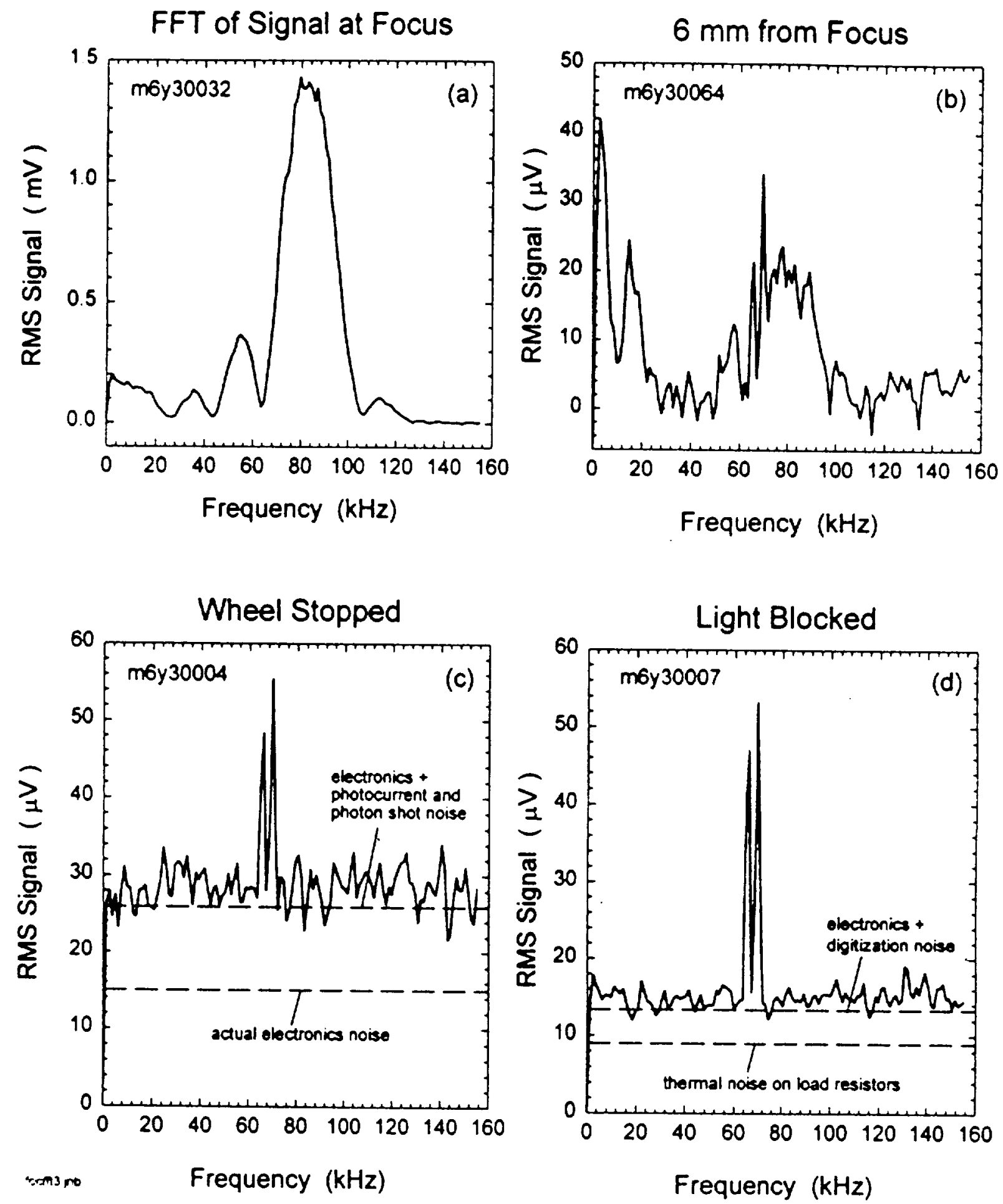

Figure 9. FFT's of rotating wheel data with system noise estimates. 

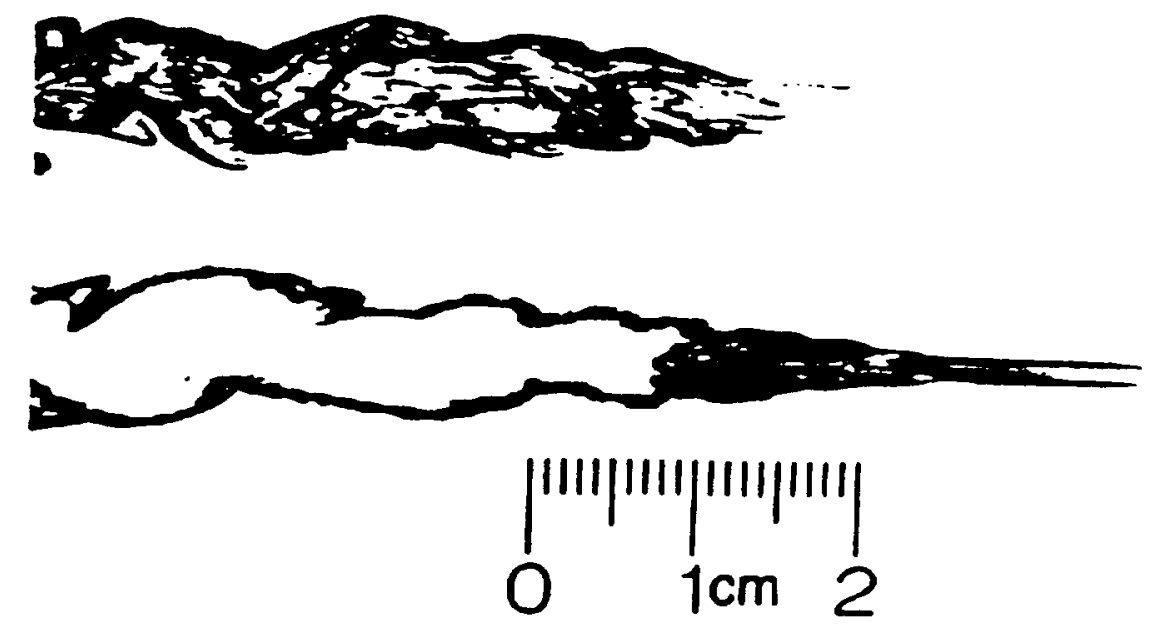

Figure 10. CCD camera images of a luminous oxy-acetylene torch flame.
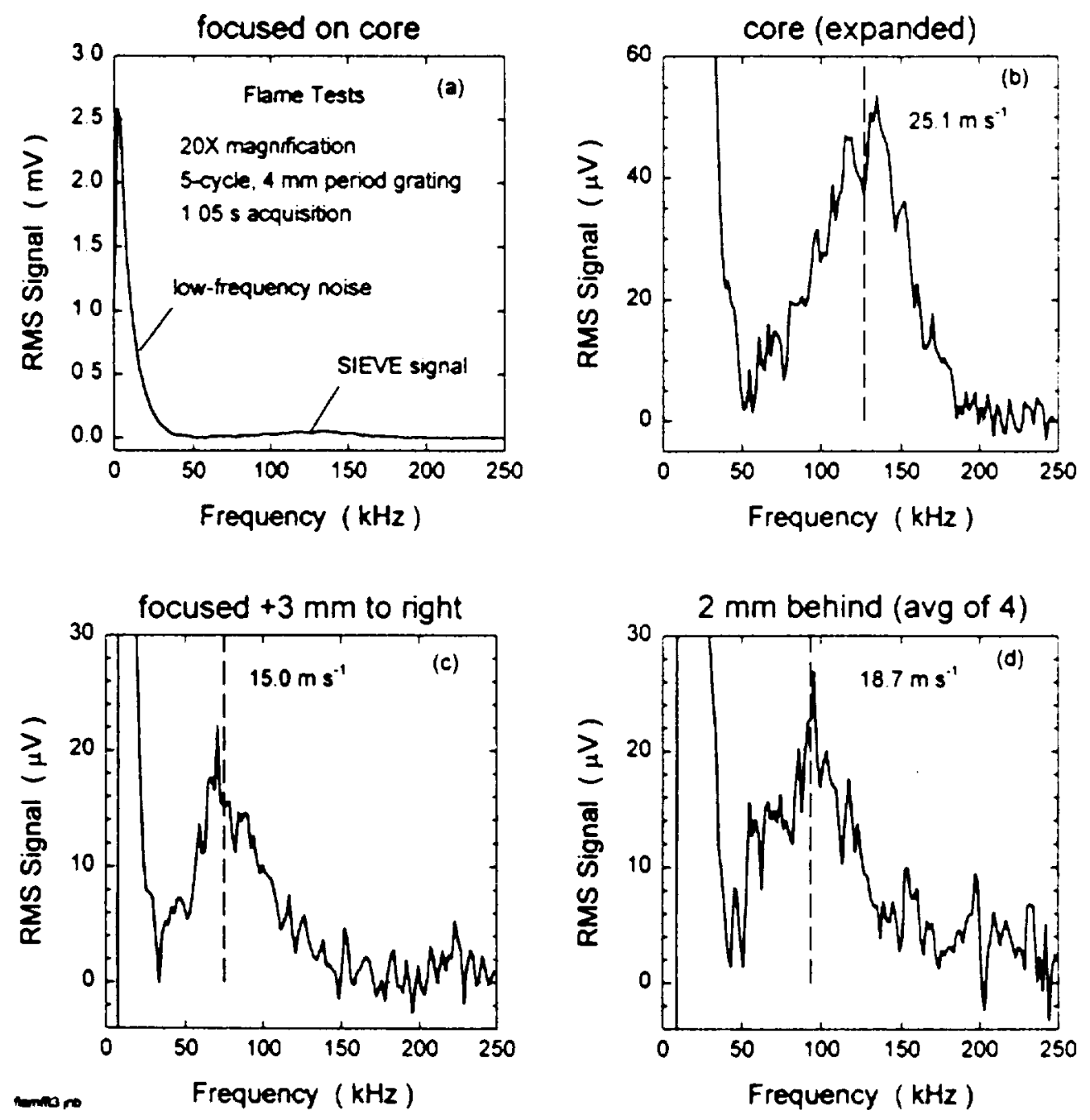

Figure 11. FFT's of luminous flame SIEVE signals. 


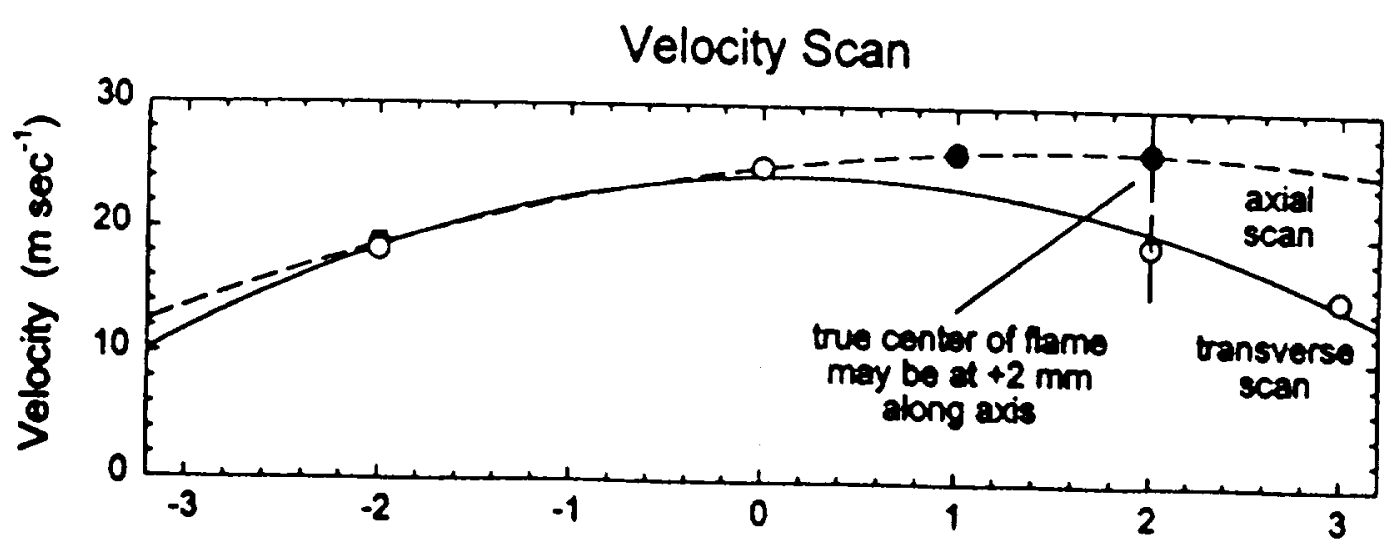

Figure 12. Axial and transverse SIEVE scans of a luminous, turbulent flame. 



\begin{tabular}{|c|c|c|c|}
\hline \multicolumn{3}{|c|}{ REPORT DOCUMENTATION PAGE } & $\begin{array}{l}\text { Form Approved } \\
\text { OMB No. 0704-0188 }\end{array}$ \\
\hline \multicolumn{4}{|c|}{ 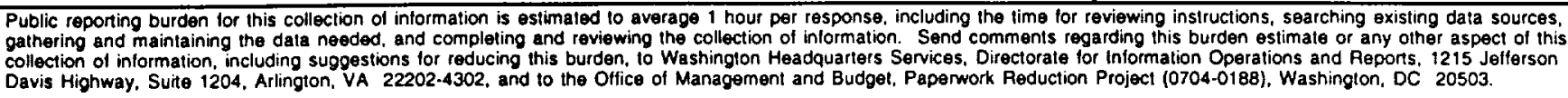 } \\
\hline 1. AGENCY USE ONLY (Leave Dlank) & $\begin{array}{r}\text { 2. REPORT DATE } \\
\text { August } 1997\end{array}$ & \multicolumn{2}{|c|}{$\begin{array}{l}\text { 3. REPORT TYPE AND DATES COVERED } \\
\text { Technical Memorandum }\end{array}$} \\
\hline \multicolumn{3}{|c|}{$\begin{array}{l}\text { 4. TITLE AND SUBTITLE } \\
\text { Achromatic Emission Velocity Measurements in Luminous Flows }\end{array}$} & \multirow{2}{*}{$\begin{array}{l}\text { 5. FUNDING NUMBERS } \\
\text { WU-632-1B-1B }\end{array}$} \\
\hline \multicolumn{3}{|c|}{$\begin{array}{l}\text { 6. AUTHOR(S) } \\
\text { S.J. Schneider, S.F. Fulghum, and P.S. Rostler }\end{array}$} & \\
\hline \multicolumn{3}{|l|}{$\begin{array}{l}\text { National Aeronautics and Space } \\
\text { Lewis Research Center } \\
\text { Cleveland, Ohio } 44135-3191\end{array}$} & $\begin{array}{l}\text { 8. PERFORMING ORGANIZATION } \\
\text { REPORT NUMBER } \\
\text { E-10865 }\end{array}$ \\
\hline \multicolumn{3}{|c|}{ 9. SPONSORING/MONITORING AGENCY NAME(S) AND ADDRESS(ES) } & $\begin{array}{l}\text { 10. SPONSORING/MONITORING } \\
\text { AGENCY REPORT NUMBER }\end{array}$ \\
\hline
\end{tabular}

\section{SUPPLEMENTARY NOTES}

Prepared for the 33rd Joint Propulsion Conference and Exhibit cosponsored by AIAA, ASME, SAE, and ASEE, Seattle, Washington, July 6-9, 1997. S.J. Schneider, NASA Lewis Research Center; S.F. Fulghum and P.S. Rostler, Science Reasearch Laboratory, Inc., Somerville, Massachusetts. Responsible person, S.J. Schneider, organization code 5430, (216) 977-7484.

12a. DISTRIBUTIONAVAILABILITY STATEMENT

12b. DISTRIBUTION CODE

Unclassified - Unlimited

Subject Categories 20 and 35

This publication is available from the NASA Center for AeroSpace Information, (301) 621-0390.

\section{ABSTRACT (Maximum 200 words)}

A new velocity measurement instrument for luminous flows was developed by Science Research Laboratory for NASA. The SIEVE (Segmented Image Emission VElocimeter) instrument uses broadband light emitted by the flow for the velocity measurement. This differs from other velocimetry techniques in that it does not depend on laser illumination and/ or light scattering from particles in the flow. The SIEVE is a passive, non-intrusive diagnostic. By moving and adjusting the imaging optics, the SIEVE can provide three-dimensional mapping of a flow field and determine turbulence scale size. A SIEVE instrument was demonstrated on an illuminated rotating disk to evaluate instrument response and noise and on an oxy-acetylene torch to measure flame velocities. The luminous flow in rocket combustors and plumes is an ideal subject for the SIEVE velocity measurement technique.

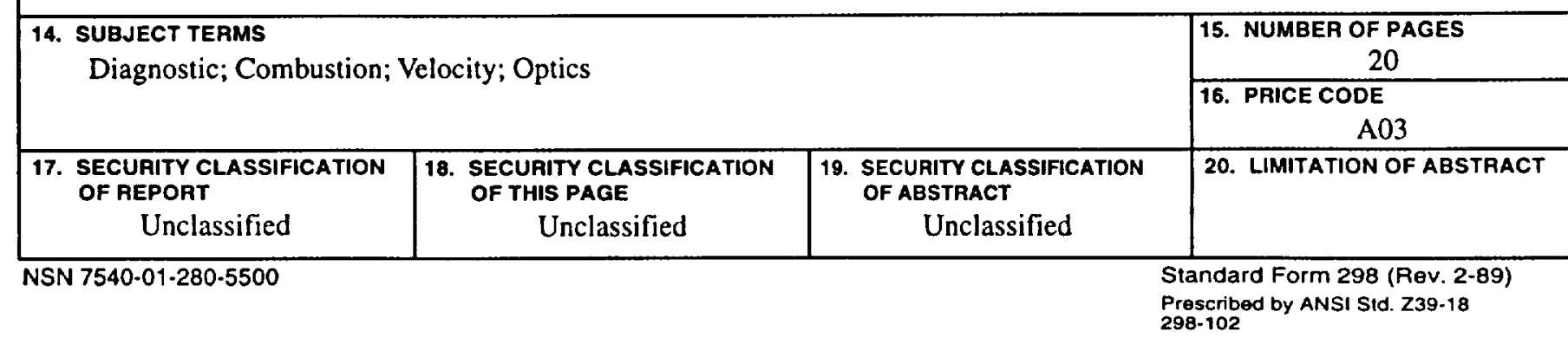

